\title{
IDMA-based cooperative partial packet recovery: principles and applications
}

Zhifeng Luo ${ }^{1 *}$, Zhu Han², Albert Kai-sun Wong ${ }^{3}$ and Shuisheng Qiu' ${ }^{1}$

\begin{abstract}
In this article, we focus on the cooperative multi-user network model and propose a relay-assisted partial packet recovery scheme in which asynchronous interleave-division multiple-access (IDMA) with iterative chip-by-chip multiuser detection (MUD) is used for the recovery of partial packets from multiple sources. In packet transmission, only a few erroneous bits may cause the entire packet to be discarded and partial packet recovery can reduce waste in resource by retransmitting only the bits that are unreliable, As the retransmitted partial packets for different sources can be of different lengths, IDMA is particularly suitable because of the simplicity of chip-by-chip MUD and because there is no need for strict synchronization. Our detailed scheme, which includes a feedback request strategy for indicating the unreliable bits, is presented and its performance is investigated. The simulation results show that the network throughput can be significantly improved by the proposed scheme, compared to traditional CDMA-based automatic repeat request (ARQ). Moreover, under the context of cognitive radio networks, we propose a hybrid strategy in which interleave division multiplexing (IDM) is used during whole-packet retransmission, and demonstrate the effectiveness of the proposed scheme with and without the hybrid strategy as well as give insights about the throughput performance under different parameter settings.
\end{abstract}

\section{Introduction}

Direct-sequence code division multiple access (DSCDMA) wireless networks are widely deployed today, such as IEEE 802.11b in [1]. At the link layer of such networks, the automatic repeat request (ARQ) protocol is often used to ensure reliable packet delivery, with cyclic redundancy check (CRC) to detect whether the received packet has errors. If any error is detected by CRC, the packet is discarded and retransmission is requested by the receiver. ARQ with a limit on the maximum number of retransmissions, called truncated ARQ, is used to limit the delay and buffer size [2]. In truncated $A R Q$, if a packet still has errors after being retransmitted for the defined maximum number of times, the packet will be discarded and a packet loss is announced. ARQ and truncated ARQ reduce the packet error rate (PER) at the expense of retransmissions.

Recently, a partial packet recovery scheme [3] is proposed for throughput improvement. In the traditional ARQ scheme, the entire packet is retransmitted even

\footnotetext{
* Correspondence: jeffman138@hotmail.com

${ }^{1}$ School of Electronic and Information Engineering, South China University of Technology, Guangzhou, China

Full list of author information is available at the end of the article
}

when only a portion of the packet has errors. The basic idea behind partial packet recovery is to retransmit only the unreliable bits if a received packet fails CRC. The case described as follows can be challenges for the existing partial packet recovery scheme: a wireless network that is under heavy load may have to handle more than one corrupted packet at the same time slot. An example of this scenario is when CSMA and RTS/CTS fail to avoid the collision between two source packets. ${ }^{\text {a }}$ If both source packets are intending for the same destination, the receiver at the destination will be required to handle the partial packet recovery for more than one packet at the same time. Hence, more than one packet need to recover at the destination in the partial packet recovery scheme. To this end, we propose to use IDMA as a partial packet recovery method, which can recover the multiple erroneous packets simultaneously.

On the other hand, cooperative transmission techniques can provide diversity gains through relays in the fading wireless channel [4-6]. This diversity gain is achieved by transmitting the source signal on multiple uncorrelated links through different relays towards the destination, and then combining the received signals for detection at the destination. In such a way, cooperative 
communication allows a source node with a single antenna to share the antennas of other nodes, resulting in a form of virtual multiple-input multiple-output (MIMO) system. Cooperative protocols include schemes such as decode-and-forward (DF) and amplify-and-forward (AF) [4-6]. In [7], a cooperative packet recovery scheme is proposed. It requires retransmission of the entire packet, and combines confidence information across multiple copies of a packet from the multiple access points that are connected by wired Ethernet. In fact, this is equivalent to a multiple antenna receiver scheme without the assistance of relay. In [8], a truncated cooperative ARQ scheme with relay-assistance is proposed in which the source and multiple relays use an orthogonal space-time block code (STBC) to retransmit an entire packet. But this scheme requires close synchronization of the source and the relays for STBC to work, and coordinating different transmitters in the wireless network can be difficult.

Interleave-division multiple-access (IDMA) [9] has the advantage that it can work without synchronization among the source and the relays, and it provides a good interference cancellation performance. Moreover, the multi-user detection (MUD) in IDMA has a linear complexity, implying a lower cost than the MMSE-based MUD that has polynomial complexity in CDMA [10-12]. In [13], a scenario is described in which multiple source-destination pairs are assisted by multiple common relays based on IDMA. The study in [13] shows that IDMA relays at different locations can provide different diversity gains for the multiple source-destination pairs. In our scheme, the proposed IDMA-based partial packet recovery integrates the cooperative transmission technique by our relay-assisted retransmission protocol. The proposed scheme inherits the advantage that IDMA MUD has the low complexity.

Cooperative communications could be particularly attractive in cognitive radio networks where secondary users are allowed to utilize the spectrum if the spectrum is not occupied by the primary users [14-16]. Hence, secondary users may be able to obtain more transmission opportunities by assisting the primary users to complete their transmissions as quickly as possible $[17,18]$. Incentive mechanisms can be used to encourage secondary users to serve as cooperative relays [19]. In [20], a cooperative ARQ scheme based on an auction mechanism to select the best secondary users as a primary user's relays is proposed for cognitive radio networks. In this scheme, the secondary users help a primary user to retransmit on the condition that the primary user reimburses them with parts of the retransmission time slots in return, making it a major concern to the secondary users how they may obtain as many transmission time slots as possible. We call the above tradeoff case as utility issue of secondary user cooperation. As we know, different from the general cooperative transmission scheme, the cooperative transmission scheme in cognitive radio networks scenario is necessary to consider utility issue of secondary user cooperation. In this article, to address the utility issue, we propose a hybrid strategy and reveal applicability of the proposed partial packet recovery scheme to cognitive radio networks. In the proposed hybrid strategy, interleave division multiplexing (IDM) [21,22], a generalization of IDMA, is a spectral efficient scheme for the secondary users to gain more transmission time slots. IDM can be easily applied into our scheme for enhancing the throughput.

The contributions in this article are as follows:

1) We propose to apply the principle of IDMA as a novel partial packet recovery method. Our scheme takes advantages of IDMA, which has low-complexity MUD and has the good performance on multiple access interference (MAI) cancellation, for recovering multiple erroneous packets simultaneously. In addition, the asynchronous property of iterative chip-by-chip MUD mechanism in our proposed IDMA scheme enables the receiver to extract the multiple partial packets of different sizes in the case of multiple partial packets recovery. Different from the reliability-based hybrid ARQ scheme proposed in [23,24], our scheme does not need to take any channel coding scheme into consideration except the repetition code which is the inevitable component integrated with interleavers in the IDMA transmitter, and requires the retransmission of only the unreliable bits instead of the coded redundancy information. Unlike the sub-packet scheme proposed in [25], in our scheme, the data packet does not have to be divided into sub-packets and does not have to be encoded by a group of encoders at the transmitter for sub-packet retransmission implementation. In addition, the number of sub-packets has to be determined beforehand in the scheme proposed in [25]. Our scheme does not require that the size of the retransmitted partial packet be determined in advance. Rather, the size of the retransmitted partial packet can be dynamically determined according to the received packet's bit error level in each retransmission. The simulation results show that the proposed scheme outperforms the traditional ARQ.

2) We combine cooperative retransmission technique with IDMA-based partial packet recovery so that diversity gains can be achieved while interference among multiple received partial packets can be canceled. The proposed scheme relaxes the synchronization requirement of existing relay-assisted STBC retransmission scheme [8].

3) We revised the cost-based evaluation method, proposed in [3], to determine the best feedback request 
strategy. Unlike the method in [3], our method does not require the calculation and storage of the cost of each possible packet chunking. Our method saves effort by using a top-down approach.

4) We give insights about the applicability of the proposed scheme in the cooperative cognitive radio network context. In our scheme, secondary users perform cooperative retransmissions as relays. To address the utility issue, a hybrid strategy is proposed, in which when it is decided that the whole packet should be retransmitted under partial packet recovery, secondary users may use IDM to shorten the time required for retransmissions so as to give themselves greater opportunities to make use of the spectrum. The throughput can be enhanced by only increasing the transmit power during whole-packet retransmissions.

This article is organized as follows: In Section 2, we introduce the system model. In Section 3, we present the proposed IDMA-based partial-packet-recovery scheme. Applicability of the proposed scheme to cognitive radio networks is discussed in Section 4. In Section 5, we show the simulation results. In Section 6, we provide a conclusion to this article.

\section{System model}

Assume that we have $K$ sources, one destination and $U$ relay nodes at different locations in a wireless communication network. Here we do not attempt to propose a new relay selection scheme, and hence we assume that the best relay for each source is known via some means. An example of efficient relay selection algorithm can be found in [20]. Also, for simplicity of illustration, we assume that each source has a distinct best relay. That is, there are $K$ best relays selected from $U$ relay candidates for assisting the recovery of erroneous packets from the $K$ sources. These relays have no error in decoding the packets from the sources, as achieved by $\mathrm{CRC}$ at the relays' receivers. If a relay receives the packets which cannot pass the CRC check, this relay will not become a candidate selected for cooperative retransmission. Figure 1 shows a linear network model with one destination $D, K=2$ sources (labeled $S_{k}, k=1,2$ ), and $U=2$ relays (labeled $R_{u}, u=1,2$ ). The roles of all nodes are assumed fixed in the network. Each node works in a half-duplex mode, and it is assumed in our analysis that BPSK is used for modulation. Also, we assume that DS-CDMA, which is the most commonly used technique in real wireless networks today, is used in the initial transmission (called Phase I below) from sources to destination. Subsequently, if retransmissions are required, the relays will use IDMA-based partial packet scheme to retransmit partial packets to the destination on behalf of the sources. The proposed scheme does not require, but also does not preclude, changes in the modulation (e.g., BPSK) and spectrum sharing (e.g., DS-CDMA) techniques used for the initial transmission. We further assume that the feedback channel is assumed to be error-free. This same assumption is made in [25]. The efficient timing and channel estimation methods for our system can be found in [26-28].

The proposed protocol operates in three phases Phase I, II, III. The whole protocol is summarized in Table 1. As we can see, the proposed IDMA-based partial packet recovery is activated only if the one or more received packets fail CRC. The signal model is given in detail as follows. In Phase I, multiple sources send their information packets to destination $D$. The relays listen and each stores the information from the source that it is assisting. The received signal at destination $D$ and relay $R_{u}$ can be represented respectively as:

$$
Y_{D}^{I}=\sum_{k=1}^{K} \sqrt{H_{S k D} P_{S k}} X_{S k}+N_{D^{\prime}}^{I}
$$

and

$$
Y_{R u}^{I}=\sqrt{H_{S k R u} P_{S k}} X_{S k}+N_{R u^{\prime}}^{I}
$$

where $H_{S k D}$ and $H_{S k R u}$ are the channel gains from source $S_{k}$ to destination $D$ and from source $S_{k}$ to relay $R_{u}$, respectively. $P_{S k}$ denotes the transmit power to destination $D$ from source $S_{k}$. $N_{D}^{I}$ and $N_{R u}^{I}$ represent the noise levels at the destination and relay $u$, respectively. $X_{S k}$ is the unit-power CDMA signal transmitted by $S_{k}$.

In Phase II, the destination checks the correctness of the received packets by CRC. If any received packet has any error, the destination requests retransmissions; otherwise, the system goes back to Phase I and any source node that has packets to send will send its next packet. Details on Phase II will be described in Section 3. In Phase III, if one or more retransmissions are requested by the destination, the retransmissions will be handled by the relays based on partial packets and

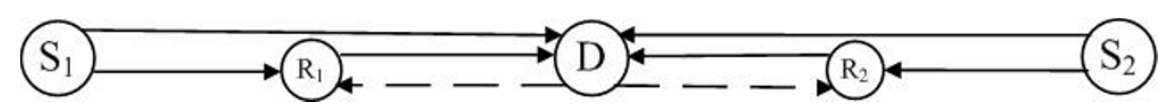

Figure 1 Relay-assisted partial packet recovery network model. The solid lines denote the data transmission between nodes, and the dashed lines denote the feedback requests from destination for retransmission. To assist $S_{1}$ and $S_{2}, R_{1}$ and $R_{2}$ are the relays to respond to the feedback request. 
Table 1 IDMA-based cooperative partial-packet-recovery protocols.

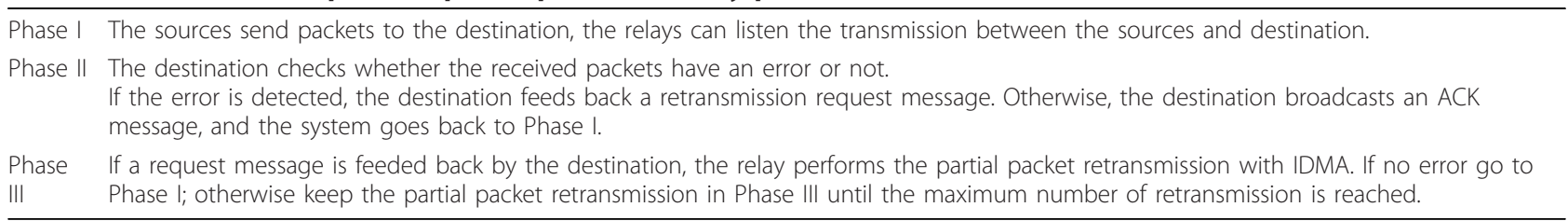

IDMA, and the received signal at the destination in this phase is given by:

$$
Y_{D}^{I I I}=\sum_{u=1}^{K} \sqrt{H_{R u D} P_{R u}} X_{R u}+N_{D}^{I I I} .
$$

We assume that the received signal is a function of discrete time instances $j$; that is, $Y_{D}^{I I I}=\left\{\gamma_{D}^{I I I}(j), j=1,2, \ldots, \max [I(u)]\right\}$, where $I(u)$ denotes the length of the partial packet transmitted by relay $R_{u}$. Also, the signal transmitted by relay $R_{u}$ is $X_{R u}=\left\{x_{R u}(j-\right.$ $\left.\left.d_{u}\right), j-d_{u}=1,2, \ldots, I(u)\right\}$, which is the unit-power signal generated by the IDMA transmitter at relay $R_{u}$, where $\left\{d_{u}, u=1,2, \ldots, K\right\}$ denotes the delay variables for different partial packets. $H_{R u D}$ is the channel gain from relay $R_{u}$ to destination $D, P_{R u}$ is the transmit power at $R_{u}$, and $N_{D}^{I I I}$ is the noise level at $D$. $N_{D}^{I I I}=\left\{n_{D}^{I I I}(j)\right\}$ follows a Gaussian distribution with variance $\sigma^{2}$.

Figure 2a shows the structure of the CDMA receiver at the destination, which is equipped with an IDMA partial recovery module. In Figure $2 \mathrm{a}$, output from the demodulation unit includes the hard decoding bits and the soft bits. The soft bits, which will be described in Section 3, we can provide information about the confidence level of each bit. The "unreliable bits detection (UBD)" block uses the confidence information to detect unreliable bits in the received packet. The destination then feeds back a retransmission request for the unreliable bits in each received packet to an appropriate relay. This retransmission request information, denoted by a list of indices of bits $R_{\text {list }}$, also input into the "unreliable bits repair" block. In Figure 2a, multiple partial packets (shown as $S 1, S 2, \ldots$ ) which may have different sizes are retransmitted by different relays and received by the partial packet receiver at destination $D$, which utilizes an iterative chip-by-chip multiuser detection (MUD) to separate them. The outputs of the partial packet receiver are the multiple partial packets after hard decoding. These partial packets will be input to the unreliable bits repair block. The function of unreliable bits repair block is just to replace the unreliable bits, indexed by $R_{\text {list }}$, in the original transmissions with the input of partial packets. Finally, the repaired packets are checked by CRC. Let $n_{r}$ denote the counter of retransmission. $N_{\text {retx }}$ denotes the maximum number of retransmission. For each retransmission, $n_{r}$ is incremented by 1 . If $n_{r}=N_{\text {retx }}$, or if $n_{r}<N_{\text {retx }}$ and no any erroneous bit is detected by $\mathrm{CRC}$, the multiple partial packets recovery are completed. If $n_{r}<N_{\text {retx }}$ and a packet fails CRC, the "CRC" block indicates the "UBD" block to put a NACK message in the feedback request for the next retransmission. Figure $2 \mathrm{~b}$ shows the structure of IDMA-based partial packet receiver. The principle of IDMA-based partial packet receiver will be detailedly presented in Section 3.

In the rest of this section, we give a brief review on IDMA iterative chip-by-chip MUD [10]. Let the received signal from $K$ users at the IDMA iterative chip-by-chip MUD receiver be represent by:

$$
r(j)=\sum_{k=1}^{K} c_{k} s_{k}(j)+n_{\text {IDMA }}(j), j=1,2, \ldots, J,
$$

where $c_{k}$ is user $S_{k}$ 's channel coefficient and $\{s k(j)\}$ is user $S_{k}$ 's IDMA transmitted signal, which is generated by first coded user $S_{k}$ 's data with a repetition code and then random interleaving of the resulted chip sequence. $J$ denotes the frame length and $n_{I D M A}(j)$ is the additive white Gaussian noise with zero mean and variance $\sigma^{2}$. We can rewrite $(4)$ as $r(j)=c_{k} s_{k}(j)+\eta_{k}(j)$, where $\eta_{k}(j)=\sum_{k^{\prime} \neq k} c_{k^{\prime}} s_{k^{\prime}}(j)+n_{I D M A}(j)$ and represents the MAI. The IDMA MUD can be performed in a chip-by-chip way because the random interleaver is used. According to the central limit theorem, $\left\{\eta_{k}(j)\right\}$ approximately follows a Gaussian distribution. The IDMA chip-by-chip MUD [10] is stated as follows: At first, IDMA MUD calculates the chip-level log-likelihood ratio (LLR) about $\left\{s_{k}\right.$ $(j)\}$. We denote this $\operatorname{LLR}$ as $\operatorname{LLR}\left(s_{k}(j)\right)$, which is given by:

$$
\operatorname{LLR}\left(s_{k}(j)\right)=\frac{2 c_{k}\left\{r(j)-E\left[\eta_{k}(j)\right]\right\}}{\operatorname{Var}\left[\eta_{k}(j)\right]},
$$

where

$$
E\left(\eta_{k}(j)\right)=\sum_{k^{\prime}=1, k^{\prime} \neq k}^{K} c_{k^{\prime}} E\left[s_{k^{\prime}}(j)\right],
$$

$\operatorname{Var}\left(\eta_{k}(j)\right)=\sum_{k^{\prime}=1, k^{\prime} \neq k}^{K}\left|c_{k^{\prime}}\right|^{2} \operatorname{Var}\left[s_{k^{\prime}}(j)\right]+\sigma^{2} \cdot\left\{E\left(\eta_{k}(j)\right)\right\}$ and $\left\{\operatorname{Var}\left(\eta_{k}(j)\right)\right\}$ give us the estimated statistic characteristics of the interference. After deinterleaving, the set of chiplevel LLR values $\left\{L L R\left[s_{k}(j)\right]\right\}$ produces the bit-level LLR by the decoder of repetition code. The bit-level LLRs can provide MUD the a priori information, which is used to update the chip-level mean and variance in MUD. Then, MUD utilizes a better statistic to refine the chip-level LLR estimation in the following iteration $[10,12]$. We would like to point out the differences 


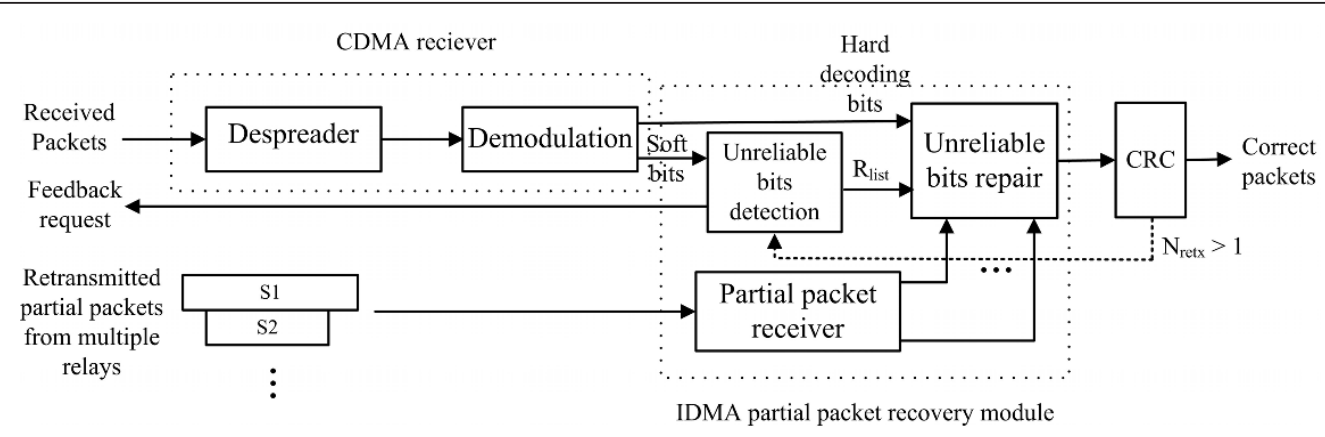

(a)

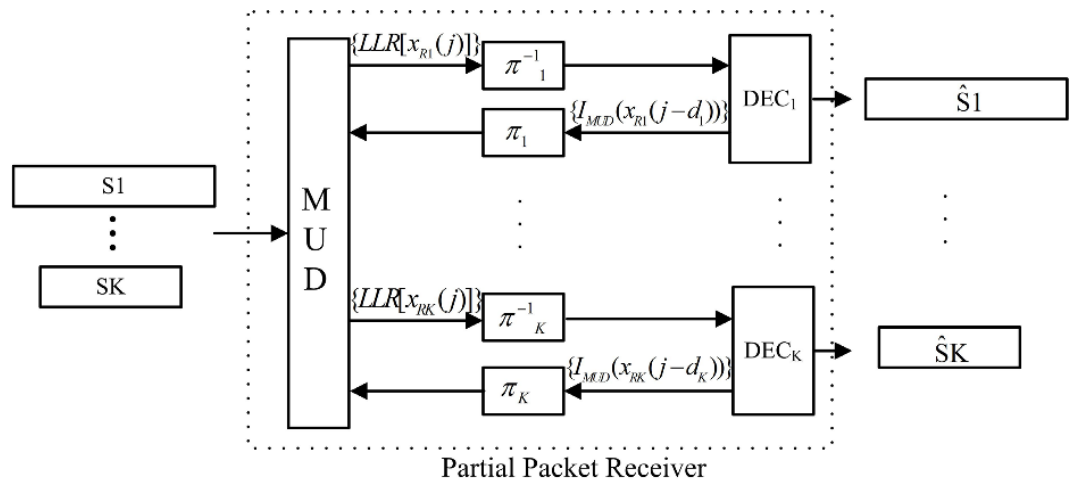

(b)

Figure 2 The structure of receiver. (a) CDMA receiver with IDMA partial-packet-recovery module. (b) The structure of partial packet recovery receiver. $R_{\text {list }}$ denotes the list of bits required to be retransmitted. $N_{\text {retx }}$ denotes the maximum number of retransmission. $n_{r}$ denotes the counter of retransmission. For each retransmission, $n_{r}$ is incremented by $1 . \pi$ and $\pi^{-1}$ the interleaver and deinterleaver, respectively. DEC denotes the decoder of repetition code.

between IDMA and CDMA as follows: IDMA uses different interleavers to separate different users which all use the same repetition code; CDMA uses different spreading sequences to separate different users. In fact, IDMA can be viewed as a special form of CDMA if the repetition code is viewed as a spreading spectrum.

\section{Partial packet recovery with IDMA method}

In our scheme, the UBD is first used to find which parts of the received packet have high error possibility in decoding. Then, according to the UBD result, a feedback request strategy is decided by the proposed recursive algorithm based on evaluation of the retransmission cost. Finally, the proposed IDMA method is used by the relays to achieve relay-assisted multiple partial packets recovery. The proposed IDMA-based partial-packetrecovery scheme is presented in detail as follows.

\subsection{Unreliable bits detection (UBD)}

For ease of discussion, we define a soft bit as a real number within $[-1,1]$. The concept of soft bits in our scheme is similar to that of soft decoding, described in
$[3,7]$. The absolute value of a soft bit indicates the confidence of decoding. The confidence value is a metric that measures the reliability in the correctness of the decoded bit. In [3,7], the confidence is calculated as the Hamming distance of the CDMA codeword for a bit. In our proposed scheme, we calculate the confidence value as a Euclidean distance. In partial packet recovery, the confidence value is forwarded up to the link layer for retransmission.

We give the mathematical expression of soft bits and confidence value as follows. Assume that the received signal $\{y\}$ is modeled by: $y(j)=h x(j)+n(j), j=1,2, \ldots$, $L$, where $x(j)$ is the CDMA transmitted signal, $n(j)$ denotes the thermal noise, $h$ is the channel coefficient. Let the transmitted BPSK symbol represented by $d(i) \in$ $\{-1,+1\}, i=1,2, \ldots, W . d(i)$ is spread by a spreading sequence $\mathbf{v}$ with the length of $V$. The spreading process is given as: $d(i) \mathbf{v} \rightarrow x(j), L=W \times V$. Let $c(i)$ denotes the output from demodulation without hard decision. To illustrate the concept, we take the first soft bit $c(1)$ as an example. After the despreading and demodulation in Figure 2, the first soft bit is given by $c(1)=\frac{\sum_{j=1}^{V} v(j) y(j)}{V}$, 
where the numerator is the summation over all chips related to the first BPSK symbol, and the denominator $V$ is for normalization. In this example, the confidence value of the first bit can be obtained by: $|c(1)|=\left|\frac{\sum_{j=1}^{V} \nu(j) y(j)}{V}\right|$.

Let $T$ denotes a preset threshold. If a bit has a confidence value, $|c|>T$, this bit is labeled as a good bit. Otherwise, this bit is labeled as a bad (unreliable) bit, and will be included in the retransmission request. As an example, a 16-bit packet with UBD is illustrated in Figure 3. The confidence value of each bit in the packet is obtained by the soft bit, which is the output from the demodulation in the physical layer. The UBD can be implemented in the link layer as suggested in [3], and the confidence information is conveyed from the physical layer to the link layer. In Figure 3, the indexes of bits with the confidence values lower than the threshold are $1,2,4,6,9,13,14$, and 16 . Only these unreliable bits are requested to be retransmitted.

\subsection{Recursive algorithm of feedback request strategy}

Different from simple ACK/NACK ARQ, partial-packetrecovery requires feedback of the indexes information of the unreliable bits. If the amount of the index information is large, the cost of the feedback request is large and the overall throughput performance can be degraded. Hence, the feedback request strategy needs to be designed carefully. We modify the cost-based method, which is originally proposed in [3], to design a recursive algorithm. Different from the method proposed in [3], our method does not use a bottom-up approach and does not calculate the cost of every possible packet chunking. The flow chart of our proposed algorithm is shown in Figure 4. First, the unreliable bits in a decoded packet are detected and the indexes of the unreliable bits are obtained. Let the set $A=m, \ldots, n^{\prime}$, . $\ldots m^{\prime}, \ldots, n$ denotes an ordered index set (i.e., $m<n^{\prime}$ $\left.<m^{\prime}<n\right)$ of a group of unreliable bits. Assume a packet has $L$ bits, then each index requires $\log _{2} L$ bits. The cost of retransmitting the entire block which contains all bits from the $m$ th position to the $n$th position in a packet is given by:

$$
C_{I}=2 \log _{2} L+n-m+1 \text {, }
$$

which includes the starting and ending index of the block and $n-m+1$ retransmitted bits. Similarly, the cost of dividing the entire block into two sub-block is obtained by:

$$
C_{I I}=2 \log _{2} L+n^{\prime}-m+1+2 \log _{2} L+n-m^{\prime}+1,
$$

where $m^{\prime}$ and $n^{\prime}$ are the new starting index and the new ending index for division from a block into two sub-blocks, respectively. We use the following criterion to select the $m^{\prime}$ and $n^{\prime}$ in a block:

$$
\max \left(m^{\prime}-n^{\prime}-1\right), \quad \text { s.t. } \quad m^{\prime}, n^{\prime} \in A, m \leq n^{\prime} \leq m^{\prime} \leq n,
$$

where $\left(m^{\prime}-n^{\prime}-1\right)$ indicates that there are $\left(m^{\prime}-n^{\prime}-1\right)$ reliable bits between the $m^{\prime}$ th and the $n^{\prime}$ th unreliable bits. In (8), the cost $C_{I I}$ can be minimized by maximizing ( $m$ ' $\left.n^{\prime}-1\right)$. In Figure $4, C_{I}$ and $C_{I I}$ are calculated in the "The calculation of retransmission cost for Input_Block" block, where Input_Block denotes the block which is the input of the calculation of retransmission cost. In other words, $C_{I}$ and $C_{I I}$ represent two options to treat the entire block: retransmission without division and retransmission with division. For the option in (7), $\left(m^{\prime}-n^{\prime}-1\right)$ reliable bits are not retransmitted, as the entire block which is from the $m$ th to the $n$th bits is divided into the left and right two sub-blocks which include the $m$ th to the $n^{\prime}$ th bits and the $m$ 'th to the $n$th bits respectively. Let $B_{\text {Left }}$ and $B_{\text {Right }}$ denotes the left sub-block and the right sub-block, respectively. As shown in Figure 4, a decision whether it is worth to divide a block into two sub-blocks is made by evaluating the cost between the two options. We select the option with a smaller cost, which can be represented by $\min \left(C_{I}\right.$, $\left.C_{I I}\right)$. If the division is decided, push $B_{\text {Right }}$ into a stack and then let Input_Block $=B_{\text {Left }}$; otherwise, output the starting index and length of the block, then pop the next block from the stack for the next iteration. For initialization, let Input_Block $=A$ in the first-run. Our recursive algorithm keeps running until the stack is empty. The starting index and length of each retransmission block are broadcasted in the feedback channel. The difference between our method and the one proposed in [3] is that our method compares the cost between the entire block retransmission and a block division retransmission excluding maximum reliable bits at each iteration instead of calculating the retransmission cost of every possible block division. When many unreliable bits uniformly scatter over the packet, the method proposed in [3] which calculates the retransmission cost of every possible sub-block will take a great deal of effort. In that case, the proposed recursive algorithm

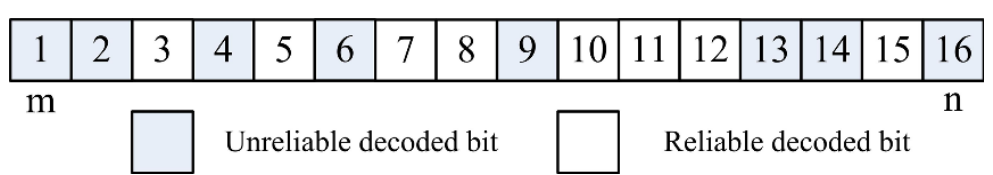

Figure 3 Unreliable decoded bits in a 16-bit packet 


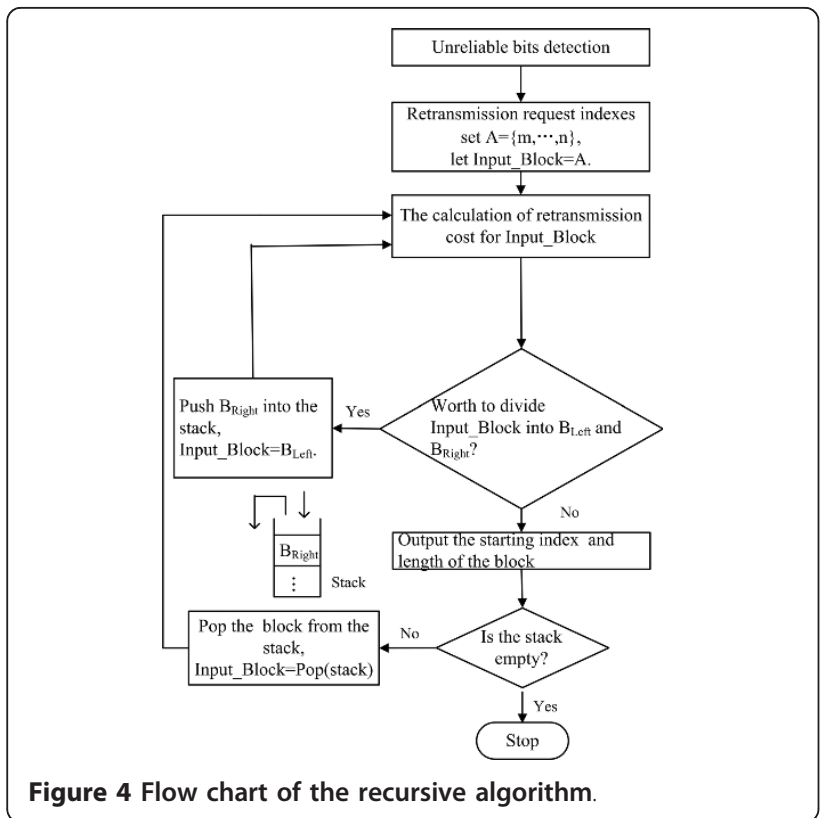

will converge to the final feedback request strategy solution after only few iterations, so our method is more easy to apply.

\subsection{IDMA in partial packet recovery}

The proposed IDMA-based partial packet recovery method is activated only if the received packet is detected to have the CRC error. The relay assists the source to retransmit the partial packets with IDMA when the proposed scheme is activated. In the case of multiple packet partial recovery, multiple relays apply the IDMA method to transmit the multiple partial packets to the destination for the recovery. In Figure 1, the IDMA partial packet receiver applies the asynchronous iterative chip-by-chip MUD to decode the multiple partial packets from the multiple relays. The log likelihood ratio (LLR) output from the asynchronous iterative chip-by-chip MUD is given as follows:

$$
L L R\left[x_{R u}(j)\right]=\frac{2 \sqrt{H_{R u D} P_{R u}}\left\{\gamma_{D}^{I I I}(j)-E\left[\eta_{R u}(j)\right]\right\}}{\operatorname{Var}\left[\eta_{R u}(j)\right]},
$$

where

$$
\begin{aligned}
& E\left[\eta_{R u}(j)\right]=E\left[\gamma_{D}^{I I I}(j)\right]-\sqrt{H_{R u D} P_{R u}} E\left[x_{R u}\left(j-d_{u}\right)\right] \\
& \left.\operatorname{Var}\left[\eta_{R u}(j)\right]=\operatorname{Var}\left[\gamma_{D}^{I I I}(j)\right]-H_{R u D} P_{R u} \operatorname{Var}\left[x_{R u}\left(j-d_{u}\right)\right] 11\right) \\
& E\left[\gamma_{D}^{I I I}(j)\right]=\sum_{u=1}^{K} \sqrt{H_{R u D} P_{R u}} E\left[x_{R u}\left(j-d_{u}\right)\right]
\end{aligned}
$$

and

$$
\operatorname{Var}\left[\gamma_{D}^{I I I}(j)\right]=\sum_{u=1}^{K} H_{R u D} P_{R u} \operatorname{Var}\left[x_{R u}\left(j-d_{u}\right)\right]+\sigma^{2} .
$$

Equation (9) detects the signal from the $u$ th relay in the multiple packets signal $\left\{\gamma_{D}^{I I I}(j)\right\}$. Equations (10) and (11) are respectively the mean and variance of the interference for the received signal from the $u$ th relay. Equations (12) and (13) are the mean and variance of the multiple packets signal, respectively. The IDMA MUD estimates the statistic of the interference iteratively. The update rule for estimation in each iteration for the $u$ th partial packet is given by:

$$
\begin{gathered}
E\left(x_{R u}\left(j-d_{u}\right)\right)= \begin{cases}\tanh \left(\frac{I_{M U D}\left(x_{R u}\left(j-d_{u}\right)\right.}{2}\right), & \text { if } 1 \leq j-d_{u} \leq I(u), \\
0, & \text { otherwise; }\end{cases} \\
\operatorname{Var}\left(x_{R u}\left(j-d_{u}\right)\right)= \begin{cases}1-E^{2}\left(x_{R u}\left(j-d_{u}\right)\right), & \text { if } 1 \leq j-d_{u} \leq I(u), \\
0, & \text { otherwise, }\end{cases}
\end{gathered}
$$

where $I_{M U D}\left(x_{R u}\left(j-d_{u}\right)\right)$ represents the a priori information provided by the decoder of repetition code for MUD in the IDMA receiver. According to Equation (3), the $u$ th relay retransmits the requested partial packet for source $u$ and the length of the partial packets is $I(u)$, which can be different for different $u$. From Equations (9) to (15), it can be seen that the proposed scheme can handle multiple partial packets with different block lengths simultaneously.

\section{Applicability to cognitive radio networks}

In this section, we apply the proposed protocol to the cognitive radio network context, and describe an optional enhancement that can be deployed by secondary users in cognitive radio networks to increase their transmission opportunities at the cost of higher transmission power. In general, cognitive radio networks have two different classes of users: primary users and secondary users. The secondary users are required not to affect the performance of primary users when they coexist in the network with the primary users. The secondary users have to sense the licensed spectrum to discover spectrum holes and avoid interfering with primary users. It is expected that with appropriate incentive mechanisms, the secondary users can be incentivized to perform as relays for primary users' ARQ retransmission [20]. For example, the secondary users, by giving assistance, can enable the primary users to release the spectrum more quickly, thus making more transmission opportunities available to themselves in return.

Our cooperative partial packet recovery model, as illustrated in Figure 1, is geared towards this cognitive 
radio network context, in which the destination $D$ is an access point that both the primary users and secondary users hope to access. $S_{1}$ and $S_{2}$ are two primary users, and $R_{1}$ and $R_{2}$ are two secondary users. $R_{1}$ and $R_{2}$ transmit their data to $D$ only when $S_{1}$ and $S_{2}$ are not transmitting over the spectrum. To be consistent with the assumption in Section 2, $R_{1}$ and $R_{2}$ are known to be the best relay for $S_{1}$ and $S_{2}$ respectively. In this article, there is no intention to develop a protocol involving incentive strategies and relay selection algorithms, and in this section we focus on the application of the proposed partial packet recovery under the assumption that the best secondary user is known. One example of the secondary user selection scheme is proposed in [20].

As we stated in Section 3.2, the proposed feedback request strategy may be required for either the whole packet retransmission or the several parts of packet retransmission. It depends on the cost evaluation of retransmission. As it is of interest to the relays, which are secondary users under the cognitive radio network context, to reduce the retransmission time for cooperation, we propose that the relays can first segment the primary user data into layers and superimpose these layers using IDM for retransmission. In this way, the secondary users can adopt the hybrid retransmission strategy, in which IDM is applied when the proposed feedback request strategy is that the whole packet is required to be retransmitted. It is known that IDM features high spectral efficiency and flexible rate adaptation [21]. An IDM-based ARQ protocol is an efficient scheme for supporting multiple QoS requirements in the point to point communication systems [22]. Let $b_{S k R u}$ denotes the $k$ th primary user's data sequence received by the $u$ th cooperative secondary user, $R_{u}$. To improve spectral efficiency, $R_{u}$ partitions $b_{S k R u}$ into several equal-length data sequences represented as $b_{u l^{\prime}}^{k} l=1, \ldots, N_{\text {layer }}^{u}$, where $N_{\text {layer }}^{u}$ is the number of layers. We call each $b_{u l}^{k}$ as a layer. Each layer is first coded with a repetition code with length $L_{S}^{u}$, subsequently interleaved with a layer-specific interleaver. The result is then modulated by BPSK to produce what we denote as $\hat{x}_{u l}^{k}(j)$. The multiple layers are linearly superimposed into the transmitted signal $X_{R u}=\left\{x_{R u}(j)\right\}, j=1,2, \ldots, L_{S}^{u} \times L_{\text {layer }}^{u}$, where $L_{\text {layer }}^{u}$ is the length of each layer's data, $L_{S}^{u}$ is the length of the repetition code. $x_{R u}(j)$ is given by:

$$
x_{R u}(j)=\frac{1}{\sqrt{N_{\text {layer }}^{u}}} \sum_{l=1}^{N_{\text {lyyer }}^{u}} \hat{x}_{u l}^{k}(j) .
$$

We can rewrite (3) with (16) to re-express the received signal at $D$ at different time index $j$ as:

$$
\gamma_{D}^{I I I}(j)=\sum_{u=1}^{K} \sum_{l=1}^{N_{\text {layer }}^{u}} \sqrt{\frac{H_{R_{u} D} P_{I D M}^{u}}{N_{\text {layer }}^{u}}} \hat{x}_{u l}^{k}\left(j-d_{u}\right)+n_{D}^{I I I}(j),
$$

where $P_{I D M}^{u}$ denotes the transmit power for IDM at $R_{u}$. Let $V_{D}:=\sum_{u=1}^{K} N_{\text {layer }}^{u}$. With $V_{D}$ different chip-level random interleavers, the receiver structure in the IDM scheme is similar to the one in the IDMA system. It is clear that the received signal $\gamma_{D}^{I I I}(j)$ can be viewed as a signal with $V_{D}$ virtual layers. The destination receiver can apply an IDMA iterative MUD to retrieve the relayed primary users' data. Let $v \leftarrow N_{\text {layer }}^{u-1}+l$ denotes the global index identifying the $l$ th layer from $R_{u}$, $l=1,2, \ldots, N_{\text {layer }}^{u} l=1,2, \ldots, N_{\text {layer }}^{u}, u=1,2, \ldots, K$. Equation (17) can be remodeled as:

$$
\gamma_{D}^{I I I}(j)=\sum_{v=1}^{V_{D}} C_{I D M}^{v} \hat{x}_{v}^{k}\left(j-\tau_{v}\right)+n_{D}^{I I I}(j),
$$

where $C_{I D M}^{v}:=\sqrt{\frac{H_{R_{u D} P_{D M}^{u}}^{u}}{N_{\text {layer }}^{u}}}$, and $\tau_{v}$ denotes the delay of the $v$ th layer. All layers from $R_{u}$ have the same $\tau_{v}$ that equals $d_{u}$. Similar to (9), the LLR's about the $v$ th layer's chips $\left\{\hat{x}_{v}^{k}(j)\right\}$ are given by:

$$
\operatorname{LLR}\left(\hat{x}_{v}^{k}(j)\right)=\frac{2 C_{I D M}^{v}\left\{\gamma_{D}^{I I I}(j)-E\left[\eta_{v}^{k}(j)\right]\right\}}{\operatorname{Var}\left[\eta_{v}^{k}(j)\right]}
$$

where $\eta_{v}^{k}(j)$ denotes the inter-layer interference. $E\left[\eta_{v}^{k}(j)\right]=\sum_{v^{\prime}=1, v^{\prime} \neq v}^{V_{D}} E\left[\hat{x}_{v^{\prime}}^{k}\left(j-\tau_{v^{\prime}}\right)\right] \quad$ and $\operatorname{Var}\left[\eta_{v}^{k}(j)\right]=\sum_{v^{\prime}=1, v^{\prime} \neq v}^{V_{D}} \operatorname{Var}\left[\hat{x}_{v^{\prime}}^{k}\left(j-\tau_{v^{\prime}}\right)\right]+\sigma^{2}$. The chiplevel LLR $\operatorname{LLR}\left[\hat{x}_{v}^{k}(j)\right]$ can generate the bit-level LLR value $L L R\left[b_{u l}^{k}\right]$ during iterations. Hard decisions are made on $L L R\left[b_{u l}^{k}\right]$ after the last iteration. Finally, All layers' decoded bits are reassembled for recovering $b_{S k R u}$.

The secondary users are concerned about how much transmit energy is left for their own data after they have participated in cooperative retransmission. To measure this factor in Section 5, we define an energy ratio $E_{S}:=\frac{\left(T_{\text {total }}-L_{S}^{u} \times L_{\text {laver }}^{u}\right) P_{R u}}{T_{\text {total }}}$, where $T_{\text {total }}$ represents the duration of an entire packet. In our scheme, the secondary users are able to flexibly configure the IDM transmission parameters, such as $N_{\text {layer }}^{u}, L_{S}^{u}$ and $P_{I D M}^{u}$. In this case, our scheme provides the secondary users another degree of freedom to keep the quality of cooperative service for the primary users.

\section{Simulation and numerical results}

To illustrate the validity of the proposed scheme, the following simulation is set up. Two sources, one destination, and two relays constitute a wireless network. The 
channels between the nodes in the network are quasistatic flat Rayleigh fading channels. The length of a data packet is 128 bits. The frame length is 1024 chips. It is assumed that there is no channel coding except the repetition code which is inevitably integrated with interleavers in the IDMA transmitter. The length of repetition code is 8 . The number of iterations at the IDMA MUD receiver is 10 . The thermal noise power is -70 $\mathrm{dBm}$. The path loss exponent is set to be 4 . The distance between the two sources and the destination is fixed at $100 \mathrm{~m}$. Let $B_{\text {correct }}$ denotes the total number of correctly received bits in the whole transmission, $B_{T}$ is the total number of transmit bits by the sources and relays, and $B_{\text {feedback }}$ is the total number of bits for feedback request in partial packet recovery. For the performance's comparison, we define the throughput of the traditional ACK/NACK ARQ scheme as $\frac{B_{\text {correct }} \text {. And the }}{B_{T}}$ throughput of our relay-assisted IDMA partial retransmission scheme as $\frac{B_{\text {correct }}}{B_{T}+B_{\text {feedback }}}$, where $B_{\text {feedback }}$ includes all information describing which set of bits are requested for retransmission. In both cases, other overhead bits for the ACK/NACK messages are ignored. Let $N_{\text {retx }}$ denotes the maximum number of retransmissions. There are two types of curves provided in our simulation: one is the upper bound of throughput for the proposed scheme; the other is the throughput where the threshold method in Section 3.1 is adopted for UBD. The upper bound curves assume that the unreliable bits are perfectly detected so that the destination has a perfect knowledge of the erroneous bits' positions in the packet. In the simulation, for the unreliable bit detection threshold, we use the values listed in Table 2 under different transmit powers on the channel between the sources and the destination. All values of threshold in Table 2 are obtained by accumulative simulations following the approach in [3] as follows: The threshold is selected by finding a confidence value that is lower than the confidence values of a majority $(70 \%-80 \%)$ of the correctly decoded bits and higher than the confidence values of a majority (70\%-80\%) of the incorrectly decoded bits as seen in the accumulative simulation results. In addition, in the simulation, the two relays are located in the middle of the respective sources and the destination.

Figure 5 shows the improvement in the throughput with the IDMA relay-assisted partial packet recovery.

\section{Table 2 Selected confidence value thresholds in the} simulations.

\begin{tabular}{lllllll}
\hline Transmit power $(\mathbf{d B m})$ & $\mathbf{0}$ & $\mathbf{5}$ & $\mathbf{1 0}$ & $\mathbf{1 5}$ & $\mathbf{2 0}$ & $\mathbf{2 5}$ \\
\hline Threshold $T\left(\times 10^{-5}\right)$ & 2.6 & 3 & 4.5 & 8 & 12.6 & 21 \\
\hline
\end{tabular}

The horizontal axis $P_{T}$ denotes the transmit power of sources and relays. It can be seen that the proposed scheme has an advantage over the traditional ARQ with whole packet retransmission. When the transmit power is $10 \mathrm{dBm}$, the proposed scheme has a throughput increase of $28 \%$, compared to traditional ARQ. When the transmit power is $5 \mathrm{dBm}$, the proposed scheme has a throughput increase of at least $55 \%$. There is a performance loss compared to the performance upper bound because there can be errors in detection of the unreliable bits using the confidence threshold.

In Figure 6, we show the PER performance of the proposed scheme versus the number of retransmissions allowed. It is shown in Figure 6 that the proposed scheme has about $8 \mathrm{dBm}$ gains on transmit power compared to the traditional ARQ in the case that PER is 0.01. Compared with the throughput performance shown in Figure 5, when the maximum number of retransmission $N_{\text {retx }}$ is increased from 1 to 2, the PER performance is improved while the throughput remains almost the same. That is, the increment in $N_{\text {retx }}$ enhances the reliability of packet delivery, but there is no impact on the throughput because the system has to spend the extra time to transmit the packet successfully.

The use of cooperative transmission in traditional ARQ will clearly also improve the throughput. In Figure 7, we compare the throughput of traditional cooperative CDMA-based ARQ with the throughput of our proposed scheme under the same setting. Figure 7 shows that our scheme still outperforms the traditional cooperative CDMA-based ARQ.

In order to show the applicability of the proposed scheme to cognitive radio networks, we simulate the proposed scheme in the cognitive radio network scenarios. Under this scenarios, we also provide the throughput performance of the traditional ARQ scheme based on CDMA for comparison. In the simulation, the secondary users are allowed to transmit only when the primary users have finished their data transmissions and retransmissions. We assume that the secondary users can perfectly sense available spectrums, and that the cooperative secondary users' transmit power is the same as the primary users'. Figure 8 shows that, in the cognitive radio networks, the proposed scheme has throughput gains compared to the traditional ARQ scheme. When the transmit power of both the primary user and the secondary user is $5 \mathrm{dBm}$, the throughput gain is about $41 \%$. These gains are obtained by cooperative retransmission and multiple partial packets recovery. The secondary users acting as relays can provide spatial diversity so that reliability of packet delivery increases. The IDMA-based partial packet recovery can save time for multiple packets' retransmission. Figure 8 shows 


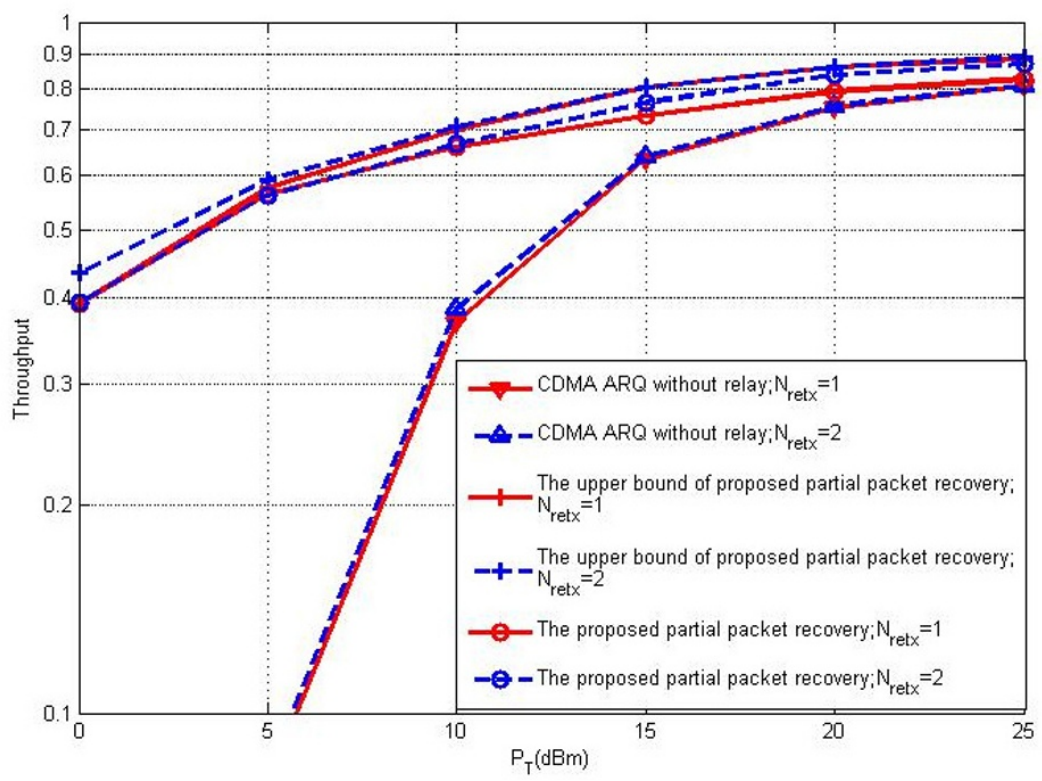

Figure 5 Throughput performance of IDMA-based partial packet recovery. $P_{T}$ denotes the transmit power of sources and relays. The distance between the sources to the destination is 100 meters. The distance between the relays to the destination is 50 meters.

that, similar to what is observed in Figure 5, in the low transmit power region, the throughput performance of the proposed scheme is close to the upper-bound.

As discussed in Section 4, when retransmitting the whole packet, the secondary users can optionally use IDM to reduce the retransmission time. Hence, on the other hand, to maintain certain quality of service requirement from the primary users when using IDM, the secondary users have to increase transmit power to compensate for the power spread over the multiple layers. Therefore, there is a tradeoff problem, and to address the above problem, we first need to find the proper IDM parameter setting, because the different IDM parameter values can affect the PER performance

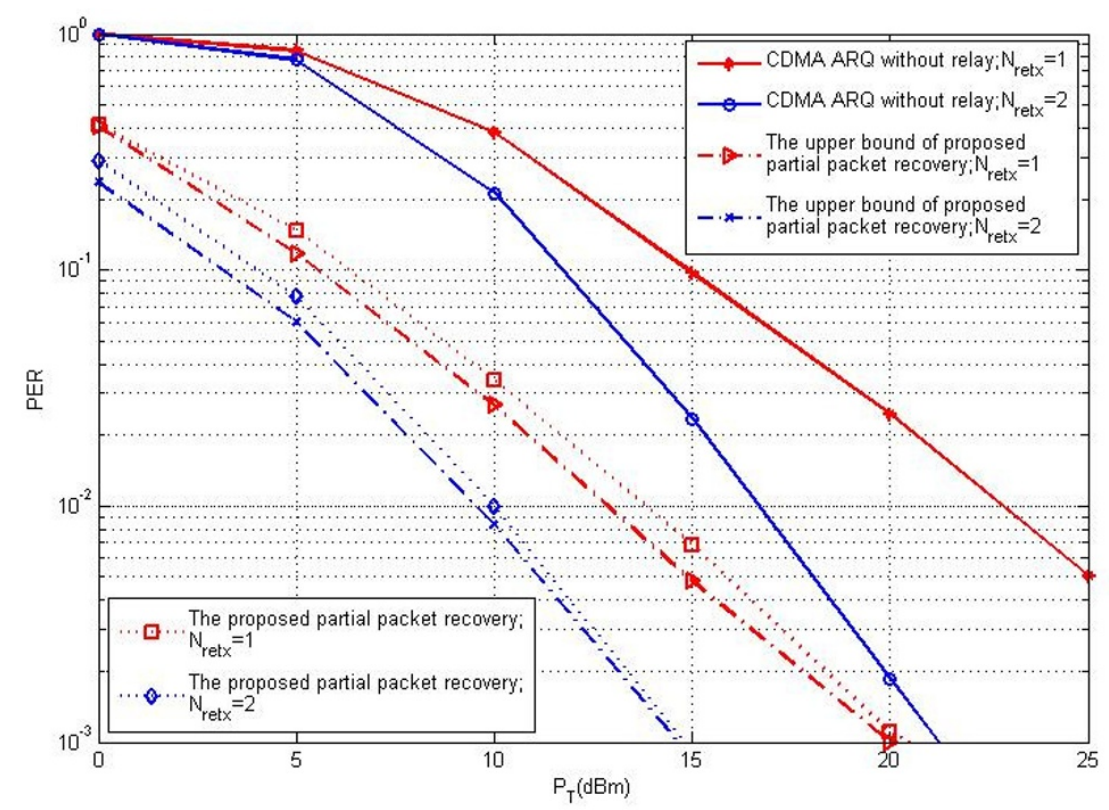

Figure 6 PER performance of IDMA-based partial packet recovery. $P_{T}$ denotes the transmit power of sources and relays. 


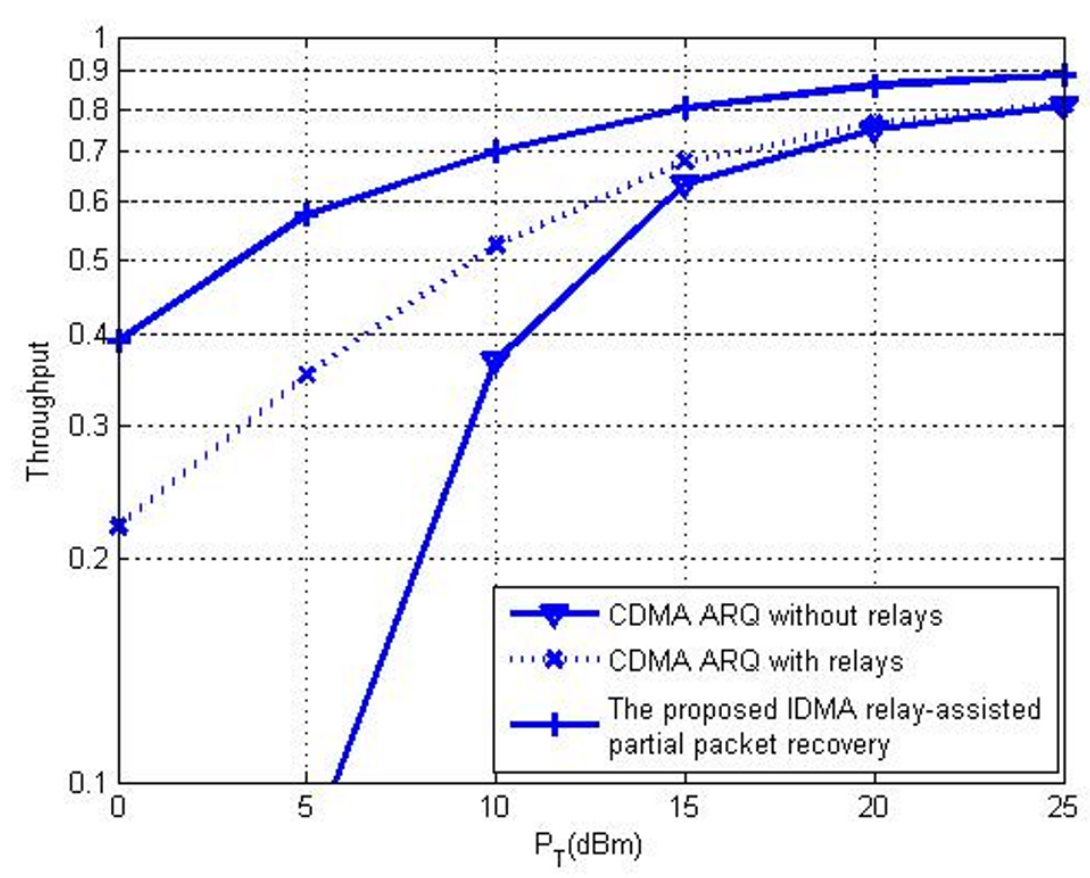

Figure 7 Comparison of throughput performance with relays. The distance between the sources to the destination is 100 meters. The distance between the relays to the destination is 50 meters.

of the retransmitted packets and the energy ratio $E_{S}$ as defined at the end of Section 4. In Figure 9, we show the PER performance under different parameter values: transmit power, the number of layers, and the length of the repetition code. In Figure 9, each distinct marker denotes a given IDM transmit power $P_{I D M}^{u}$, and each distinct line style denotes a given setting of $N_{\text {layer }}^{u}$ and $L_{S}^{u}$, represented in the form of $\left(N_{\text {layer }}^{u} L_{S}^{u}\right)$ in the figure; for

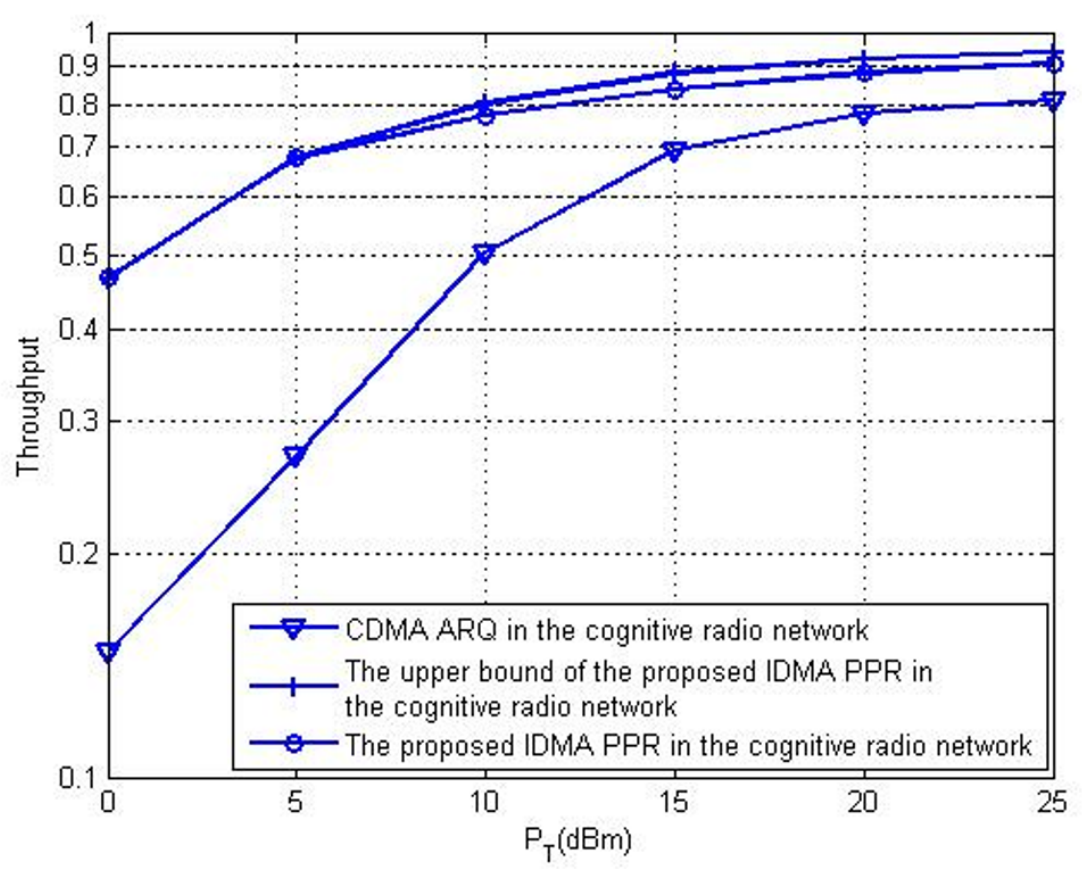

Figure 8 Throughput performance of IDMA-based cooperative partial packet recovery in the cognitive radio network. The distance between the secondary users and the destination is $\mathbf{5 0}$ meters. $N_{\text {retx }}=1$. 
example, $(2,8)$ in Figure 9 means $N_{\text {layer }}^{u}=2$ and $L_{S}^{u}=8$. The scheme that moves closer to the right-bottom of Figure 9 gives a better performance. This means that the secondary users can transmit data with a lower PER, while they spend less transmit energy for the cooperation. It is shown that scheme $(4,8)$ and scheme $(8,16)$ can result in the desirable performance when $P_{I D M}^{u}=35 \mathrm{dBm}$. For scheme (4, 8), $E_{S}=\frac{(1024-8 \times 32) \times 35}{1024}=26.25(\mathrm{~mJ})$. Compared to scheme $(8,16)$, scheme $(4,8)$ divides data into more layers but uses shorter repetition codes. Moreover, scheme $(4,8)$ and scheme $(8,16)$ have similar PER performances with the same $E_{S}$. Hence, we focus on scheme $(4,8)$ in the simulation in Figure 10.

Figure 10 shows that throughput gain can be achieved by only increasing the transmit power for the whole packet retransmission when the secondary users use the hybrid strategy for retransmission. Therefore, our proposed scheme can be considered as a power saving cooperative strategy for the secondary users. In Figure 10 , the horizontal axis $P_{T}$ denotes the power values of both the primary users' transmission and the secondary users' partial packet retransmission, and let $P_{W}$ denotes the transmit power for the whole packet retransmission. In the case of the IDM-based whole packet retransmission, $P_{W}$ is equivalent to $P_{I D M}^{u}$. The dotted line in the figure shows the throughput performance in the case where the secondary users use an equal transmit power for both the IDM-based whole packet and partial packet retransmission. The other lines indicate the throughput performance when the transmit power during whole packet retransmission is increased by the step of $5 \mathrm{dBm}$ over the transmit power during partial packet retransmission. Figure 10 shows that the throughput performance of the proposed scheme can be further improved by adjusting the power used during the whole packet retransmission relative to the power used during partial packet retransmission. Figure 10 also shows that when the transmit power during whole packet retransmission is increased to $15 \mathrm{dBm}$ to $25 \mathrm{dBm}$ above the transmit power during partial packet retransmission, no further improvement can be gained.

\section{Conclusion}

A relay-assisted partial-packet-recovery scheme using IDMA is proposed in this article. We use relays to provide diversity gain for retransmitting partial packets. The relay-assisted partial packet recovery is activated only when errors are detected in the received packet at the destination. In our scheme, multiple relays can assist to recover multiple source packets using IDMA. The asynchronous IDMA iterative chip-by-chip MUD is used by the destination to decode the multiple partial packets. Further, we adopt the concept of confidence value for the detection of unreliable bits in a packet, and the threshold detection method is introduced. To minimize the feedback request overhead, we design a

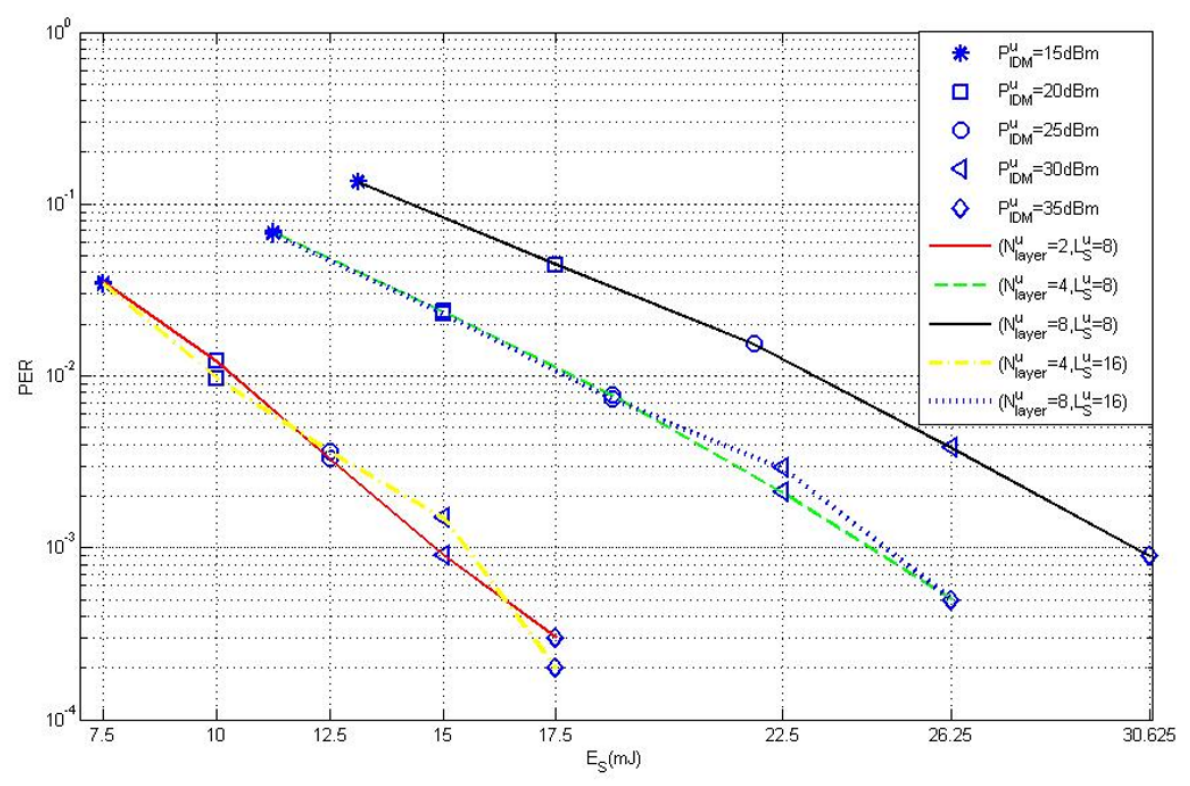

Figure 9 PER performance for different IDM settings by secondary users. Each distinct marker denotes a given IDM transmit power $P_{I D M}^{u}$ each distinct line style denotes a given setting of $N_{\text {layer }}^{u}$ and $L_{S^{\prime}}^{u}$ represented in the form of $\left(N_{\text {layer }}^{u} L_{S}^{u}\right)$ in the figure; for example, $(2,8)$ means $N_{\text {layer }}^{u}=2$ and $L_{S}^{u}=8$. The distance between the secondary users and the destination is 50 meters. 


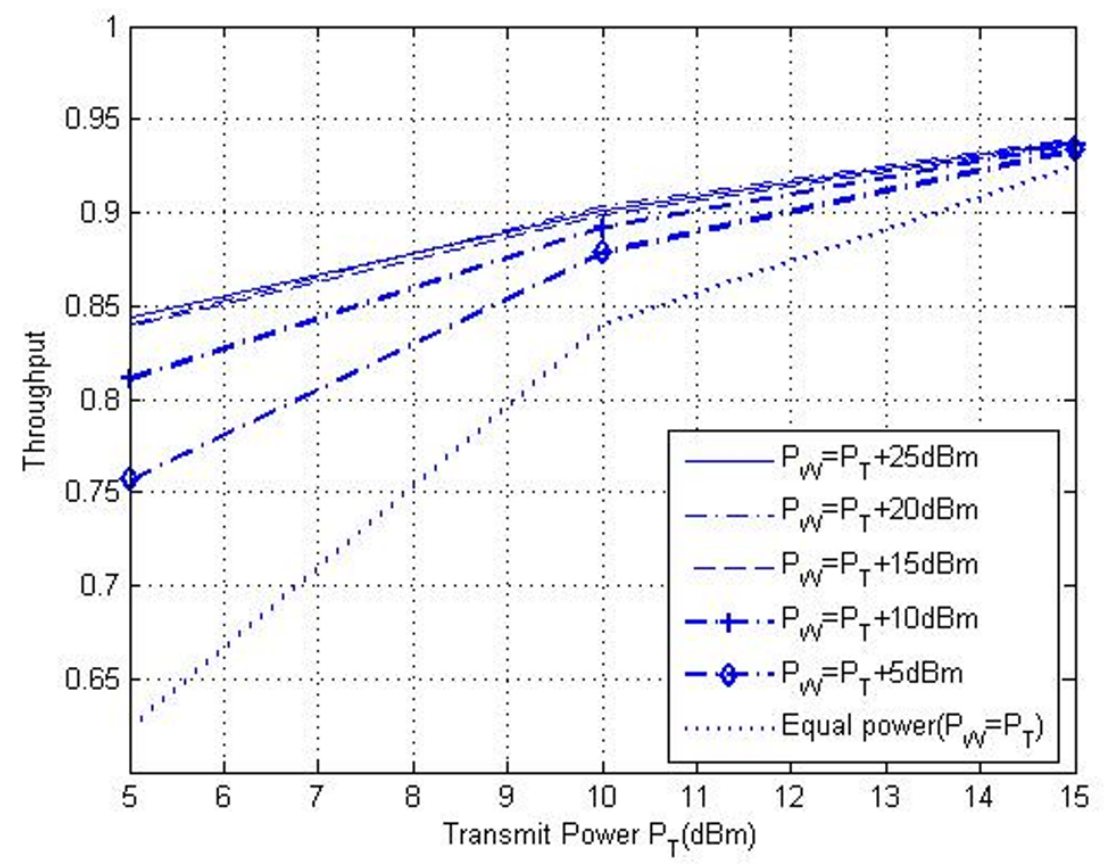

Figure 10 Throughput performance for different transmit powers $P_{w} \cdot N_{\text {retx }}=1, N_{\text {layer }}^{u}=4$, and $L_{S}^{u}=8$.

recursive algorithm based on cost evaluation to determine the retransmission strategy. Simulation results demonstrate the performance improvement of the proposed scheme over the traditional ARQ in wireless CDMA networks. Results also show that our proposed scheme performs close to the throughput upper bound. Compared to traditional ARQ, the proposed scheme has a throughput increase of approximately $28 \%$ when the transmit power is $10 \mathrm{dBm}$ and at least $55 \%$ when the transmit power is $5 \mathrm{dBm}$. In addition, we discuss the applicability of our scheme to cognitive radio networks, and show that the proposed scheme still outperforms the traditional ARQ in the cognitive radio network context. The throughput gain is about $41 \%$ when the transmit powers of both the primary user and the secondary user are $5 \mathrm{dBm}$. Moreover, the throughput performance of the proposed scheme can be further enhanced when the secondary users apply IDM to retransmit the whole packet.

\section{Endnote}

${ }^{a}$ For example, due to the hidden terminal problem.

\section{Acknowledgements}

This study was partially supported by US NSF CNS-0953377, CNS-0905556, CNS-0910461, and ECCS-1028782.

\section{Author details}

${ }^{1}$ School of Electronic and Information Engineering, South China University of Technology, Guangzhou, China ${ }^{2}$ Electrical and Computer Engineering Department, University of Houston, Houston, TX, USA ${ }^{3}$ Department of Electronic and Computer Engineering, Hong Kong University of Science and Technology, Hong Kong, China

\section{Competing interests}

The authors declare that they have no competing interests.

Received: 19 August 2011 Accepted: 9 January 2012

Published: 9 January 2012

\section{References}

1. ISO/IEC, Wireless LAN medium access control (MAC) and physical layer (PHY) specifications, ANSI/IEEE Std 802.11 (1999)

2. E Malkamaki, H Leib, Performance of truncated type-II hybrid ARQ schemes with noisy feedback over block fading channels. IEEE Trans Commun. 48(9), 1477-1487 (2000). doi:10.1109/26.870015

3. K Jamieson, $\mathrm{H}$ Balakrishnan, PPR: Partial packet recovery for wireless networks. in Proc of ACM SIGCOMM, Kyoto, Japan 409-420 (2007)

4. JN Laneman, DNC Tse, GW Wornell, Cooperative diversity in wireless networks: Efficient protocols and outage behavior. IEEE Trans Inf Theory. 50(12), 3062-3080 (2003)

5. A Sendonaris, E Erkip, B Aazhang, User cooperation diversity part I: System description. IEEE Trans Commun. 51(11), 1927-1938 (2003). doi:10.1109/ TCOMM.2003.818096

6. KJR Liu, AK Sadek, W Su, A Kwasinski, Cooperative Communications and Networking, (Cambridge University Press, Cambridge, UK, 2008)

7. GR Woo, P Kheradpour, D Shen, D Katabi, Beyond the bits: cooperative packet recovery using physical layer information, in ACM MobiCom'07, (Montréal, Québec, Canada, 2007), pp. 147-158

8. L Dai, KB Letaief, Throughput maximization of ad-hoc wireless networks using adaptive cooperative diversity and truncated ARQ. IEEE Trans Commun. 56(11), 1907-1918 (2008) 
9. Z Fang, L Li, Z Wang, An interleaver-based asynchronous cooperative diversity scheme for wireless relay networks, in IEEE International Conference on Communications, (Beijing, China, 2008), pp. 4988-4991

10. L Ping, L Liu, KY Wu, WK Leung, Interleave division multiple access. IEEE Trans Wirel Commun. 5(4), 938-947 (2006)

11. L Liu, L Ping, A comparative study on low-cost multiuser detectors, in IEEE International Conference on Communications, (Istanbul, Turkey, 2006), pp. 4947-4952

12. WK Leung, LH Liu, L Ping, Interleaving-based multiple access and iterative chip-by-chip multiuser detection. IEICE Trans Commun. E86-B(12), 3634-3637 (2003)

13. Z Luo, D Gurkan, Z Han, AK Wong, S Qiu, Cooperative communication based on IDMA, in Proceedings of the 5th International Conference on Wireless Communications, Networking and Mobile Computing, (Beijing, China, 2009)

14. E Hossain, D Niyato, Z Han, Dynamic Spectrum Access in Cognitive Radio Networks, (Cambridge University Press, Cambridge, UK, 2009)

15. S Haykin, Cognitive radio: brain-empowered wireless communications. IEEE J Sel Areas Commun. 23(2), 201-220 (2005)

16. IF Akyildiz, W-Y Lee, MC Vuran, S Mohanty, Next generation/dynamic spectrum access/cognitive radio wireless networks: a survey. Comput Networks. 50, 2127-2159 (2006). doi:10.1016/j.comnet.2006.05.001

17. AK Sadek, KIR Liu, A Ephremides, Cognitive multiple access via cooperation: Protocol design and performance analysis. IEEE Trans Inf Theory. 53(10), 3677-3696 (2007)

18. Q Zhang, J Jia, J Zhang, Cooperative relay to improve diversity in cognitive radio networks. IEEE Commun Mag. 47, 111-117 (2009)

19. O Simeone, Y Bar-Ness, U Spagnolini, Stable throughput of cognitive radios with and without relaying capability. IEEE Trans Commun. 55(12), 2351-2360 (2007)

20. I Stanojev, O Simeone, U Spagnolini, Y Bar-Ness, RL Pickholtz, Cooperative ARQ via auction-based spectrum leasing. IEEE Trans Commun. 58(6), 1843-1856 (2010)

21. H Schoeneich, PA Hoeher, Adaptive interleave-division multiple access - A potential air interface for $4 \mathrm{G}$ bearer services and wireless LANs, in Proc 1st IFIP Int Conf on Wireless and Optical Communications and Networks (WOCN 2004), (Muscat, Oman, 2004), pp. 179-182

22. PA Hoeher, H Schoeneich, JC Fricke, Multi-layer interleave-division multiple access: theory and practice. Europ Trans Telecommun. 19, 523-536 (2008). doi:10.1002/ett.1267

23. JM Shea, Reliability-based hybrid ARQ. IEE Electron Lett. 38(13), 644-645 (2002). doi:10.1049/el:20020448

24. A Roongta, JM Shea, Reliability-based hybrid ARQ and rate-compatible punctured convolutional codes, in Proc 2004 IEEE Wireless Commun and Networking Conf, vol. 4. (Atlanta, GA, 2004), pp. 2105-2109

25. Y Zhou, J Wang, Optimum Subpacket Transmission for Hybrid ARQ Systems. IEEE Trans Commun. 54, 934-942 (2006)

26. X Zhou, Z Shi, MC Reed, Iterative Channel Estimation for IDMA Systems in Time-Varying Channels, in Proc of IEEE GLOBECOM 2007, (Washington, DC, 2007), pp. 4020-4024

27. H Schoeneich, PA Hoeher, Iterative Pilot-Layer Aided Channel Estimation with Emphasis on Interleave-Division Multiple Access Systems. EURASIP J Appl Signal Process. 2006, 1-15 (2006)

28. B Senanayake, MC Reed, Z Shi, Iterative timing recovery for IDMA receivers operating under severe timing drift, in Proc of Australian Communications Theory Workshop (AusCTW), (Canberra, ACT, 2010), pp. 71-76

doi:10.1186/1687-1499-2012-2

Cite this article as: Luo et al: IDMA-based cooperative partial packet recovery: principles and applications. EURASIP Journal on Wireless

Communications and Networking 2012 2012:2.

Submit your manuscript to a SpringerOpen ${ }^{\mathcal{O}}$ journal and benefit from:

- Convenient online submission

- Rigorous peer review

- Immediate publication on acceptance

- Open access: articles freely available online

- High visibility within the field

- Retaining the copyright to your article

Submit your next manuscript at $\gg$ springeropen.com 